Check for updates

Cite this: RSC Adv., 2017, 7, 34012

Received 26th May 2017

Accepted 16th June 2017

DOI: $10.1039 / \mathrm{c} 7 \mathrm{ra05924h}$

rsc.li/rsc-advances

\section{Improved performance of HZSM-5 for the ethylbenzene/xylene isomerization reaction under industrial operating conditions}

\author{
Shokoufeh Hosseinieh Farahani, ${ }^{a}$ Seyed Mehdi Alavi ${ }^{\star a}$ and Cavus Falamaki (D) *bc \\ A mild, specific one-step dealumination of ZSM-5 is reported for the first time that significantly increases \\ para xylene selectivity and ethyl benzene conversion by disproportionation in the xylene isomerization \\ reaction of xylene mixtures/ethyl benzene feeds under industrial conditions, i.e., $380{ }^{\circ} \mathrm{C}, 390{ }^{\circ} \mathrm{C}$ and \\ $400{ }^{\circ} \mathrm{C}$, at a pressure of 8 bar, weight hourly space velocity (WHSV) values of 2 to $8 \mathrm{~h}^{-1}$, and a $\mathrm{H}_{2} / \mathrm{HC}$ \\ ratio of 4 . Oxalic acid and ammonium hexafluorosilicate (AHFS) were used as dealuminating agents. Mild \\ increases of the Si/Al molar ratio by one unit using oxalic acid or by 10 units using AHFS resulted in \\ a more than $10 \%$ increase in para xylene when approaching equilibrium at $400{ }^{\circ} \mathrm{C}$ and WHSV $=2 \mathrm{~h}^{-1}$. \\ However, the oxalic acid-treated sample increased ethylbenzene conversion by more than $30 \%$ with \\ respect to the parent zeolite under the applied operating conditions. The AHFS-treated samples \\ exhibited decreases in ethylbenzene conversion irrespective of the extent of dealumination.
}

\section{Introduction}

Despite the large number of investigations that have emerged in recent decades, there are few reports on the effects of ZSM-5 dealumination on the catalytic behavior of xylene isomerization reactions. Kumar et al. studied the influence of mild dealumination of H-ZSM-5 using $\mathrm{HCl}$, acetylene and ammonium hexafluorosilicate (AHFS) on the para xylene selectivity of meta xylene isomerization. ${ }^{1}$ They observed an increase in meta xylene conversion; however, all their dealuminated samples showed decreases in para xylene selectivity compared to the parent zeolite. A detailed study by Fernandez et al. reported the shape selectivity of hierarchical ZSM-5 zeolites for ortho xylene isomerization. ${ }^{2}$ In their work, ZSM-5 was first desilicated and consequently treated with $\mathrm{HCl}$. The resulting zeolite had lower selectivity versus para xylene with respect to the parent zeolite. Ding et al. reported increased para-selectivity for hydrothermally dealuminated ZSM-5 zeolite for the toluene alkylation reaction; however, they did not study xylene isomerization. ${ }^{3}$ Rachwalik et al. reported significant enhancement in the paraselectivity of the meta xylene isomerization reaction through a single-step dealumination treatment of a ferrierite-type zeolite (not ZSM-5). ${ }^{4}$ Detailed studies by Mueller et al. investigated the

${ }^{a}$ Chemical Engineering Department, Iran University of Science Technology, Tehran, Iran.E-mail: Alavi.m@iust.ac.ir; Fax: +98 21 77240495; Tel: +98 2177240496 ${ }^{b}$ Chemical Engineering Department, Amirkabir University of Technology, P.O. Box 15875-4413, Tehran, Iran. E-mail: c.falamaki@aut.ac.ir; Fax: +98 21 66405847; Tel: +982164543160

${ }^{c}$ Petrochemical Center of Excellence, Amirkabir University of Technology, P.O. Box 15875-4413, Tehran, Iran effects of ZSM-5 dealumination using different concentrations of oxalic acid and zeolites with different initial $\mathrm{Si} / \mathrm{Al}$ molar ratios from 9 to $46 .^{5}$ They monitored the dealumination process by ${ }^{27} \mathrm{Al},{ }^{29} \mathrm{Si}$ and ${ }^{1} \mathrm{H}$ MAS NMR together with nitrogen adsorption/ desorption analysis. While they succeeded in dealuminating the different parent zeolites by this method, the catalytic behavior of the product samples was not studied.

One of the aims of the present work is to investigate the possibility of enhancing para xylene selectivity in xylene isomerization reactions through controlled dealumination treatment of ZSM-5 zeolite by simple contact with oxalic acid or ammonium hexafluorosilicate in the liquid phase.

To our knowledge, no investigation has been reported of the effects of ZSM-5 dealumination on the isomerization reaction of xylene mixture/ethylbenzene feeds. Our attention is focused on lean para xylene mixtures. In the petrochemical industry, ethylbenzene poses a true engineering challenge in xylene isomerization plants. An economic trade-off exists between ethylbenzene minimization (complete conversion desired) through dealkylation reactions and ethylbenzene isomerization towards high para xylene selectivity. ${ }^{6}$ The former goal may be achieved using zeolites that promote ethylbenzene disproportionation reactions, and the latter may be achieved using bifunctional catalysts (e.g. Pt/H-ZSM-5). It is well known that ZSM-5 zeolite itself is effective in the transformation of ethylbenzene into ortho, meta and para xylenes; it was patented 43 years ago by the Mobil Oil company. ${ }^{7}$ However, recent industrial improvements in xylene isomerization have mostly focused on reducing the zeolite crystallite size, as disclosed in a patent by Sud-Chemie Ag. ${ }^{8}$ The same methodology, coupled with the addition of metals such as Pt, has been adopted by Mobil Oil in 
order to enhance ethylbenzene isomerization. ${ }^{9}$ A survey of relevant patents shows that specially dealuminated ZSM-5 zeolite has not been used in the xylene isomerization process.

Defect-free H-ZSM-5 is a medium pore zeolite that can enable ethylbenzene disproportionation to some extent due to the existence of inherent large cavities at the intersection of its channels. Altering the micropore architecture of ZSM-5 zeolite may influence ethylbenzene disproportionation and even change the relevant reaction mechanism..$^{10}$ At the same time, dealumination usually modifies the strength distribution and total number of acid sites, eventually altering the pore microstructure.

The present work investigates the effects of dealumination of H-ZSM-5 zeolite with oxalic acid (mild dealumination) and AHFS (mild to intense dealumination) on its physical characteristics and the performance of the dealuminated samples as catalysts in the isomerization reaction of xylene mixture/ ethylbenzene under industrial operational conditions.

\section{Experimental}

\subsection{Catalyst preparation}

An Iranian high alumina H-ZSM-5 zeolite $(\mathrm{Si} / \mathrm{Al}=18)$ with low $\mathrm{Na}_{2} \mathrm{O}$ content $(<0.08 \mathrm{wt} \%)$ from the Pars Pigment and Catalyst Co., Iran, was used. It is polycrystalline, with an average size of ca. $0.5 \mu \mathrm{m}$.

Two dealumination reagents were used, i.e. oxalic acid and ammonium hexafluorosilicate $\left(\left(\mathrm{NH}_{4}\right)_{2} \mathrm{SiF}_{6}\right.$, abbreviated as AHFS), both from Sigma-Aldrich.

The initial zeolite was dealuminated by the following procedure: (a) a proper amount of zeolite was chosen and placed in contact with an oxalic acid or ammonium fluorosilicate solution of predetermined molarity with a solid to liquid mass ratio of 1 to 10. (b) Dealumination was performed under gentle agitation at $70^{\circ} \mathrm{C}$ for a predetermined time. (c) The final product was isolated and washed with large amounts of deionized warm water to achieve a final pH close to 7. (d) The final zeolite was dried at $90{ }^{\circ} \mathrm{C}$ overnight. All zeolite samples were calcined at $c a .400{ }^{\circ} \mathrm{C}$ prior to catalytic tests.

\subsection{Characterization}

X-ray diffraction spectra of the parent and dealuminated samples were acquired using a PW1800 (Philips) instrument. The chemical compositions of the samples were determined by X-ray diffraction analysis using a PW1480 (Philips) apparatus. High-resolution transmission electron microscopy (HRTEM) was performed using a FEI Tecnai F20 instrument. Fourier transform infrared analysis (FTIR) was performed using a Spectrum GX apparatus. Adsorption/desorption isotherms for nitrogen were obtained using a 3Flex (Micromeritics) instrument. Ammonia temperature programmed desorption $\left(\mathrm{NH}_{3}{ }^{-}\right.$ TPD) experiments were performed using a Chemisorb 2750 (Micromeritics) instrument. For these tests, each sample was initially de-gassed by purging with helium gas at $150^{\circ} \mathrm{C}$ for $2 \mathrm{~h}$ with a flow rate of $20 \mathrm{~cm}^{3} \mathrm{~min}^{-1}$. Afterwards, the sample was cooled to $100{ }^{\circ} \mathrm{C}$ (still under helium purge). Then, a gas consisting of a mixture of $5 \mathrm{~mol} \%$ ammonia and $95 \mathrm{~mol} \%$ helium gas was purged over the sample for $40 \mathrm{~min}$ at the same temperature with a flow rate of $40 \mathrm{~cm}^{3} \mathrm{~min}^{-1}$. Physisorbed ammonia was egressed by consequent flow of a helium stream over the sample at $100^{\circ} \mathrm{C}$. As a final step, the sample was placed under a heating ramp of $10{ }^{\circ} \mathrm{C} \mathrm{min}^{-1}$ up to a temperature of $1000^{\circ} \mathrm{C}$ under a helium stream purge with a flow rate of $40 \mathrm{~cm}^{3}$ $\min ^{-1} \cdot{ }^{27} \mathrm{Al}$ isotope mass nuclear magnetic resonance $\left({ }^{27} \mathrm{Al} \mathrm{MAS}\right.$ NMR) spectra were obtained using a Bruker Avance III $400 \mathrm{WB}$ instrument. Thermogravimetric analysis (TGA) was performed using a Diamond TG/DTG apparatus.

\subsection{Isomerization tests}

The xylene mixture was provided by Bouali Petrochemical Co., Mahshahr, Iran. It consisted of $14.1 \mathrm{wt} \%$ ethylbenzene, $3.7 \mathrm{wt} \%$ para xylene, $54.3 \mathrm{wt} \%$ meta xylene, $24.7 \mathrm{wt} \%$ ortho xylene, 0.1 wt $\%$ toluene, and $3.1 \mathrm{wt} \% \mathrm{C}_{9}{ }^{+}$. Fig. 1 illustrates the flow diagram of the reaction setup used for the isomerization studies. A high pressure liquid pump transfers the liquid feed from the xylene mixture vessel to the T-junction, where it encounters the pressurized hydrogen gas. The ratio of hydrogen to hydrocarbon $\left(\mathrm{H}_{2} / \mathrm{HC}\right)$ is adjusted by changing the hydrogen gas flow rate with a mass flow controller $\left(\mathrm{H}_{2} / \mathrm{HC}=4\right)$. The twophase stream passes through an evaporator, where the liquid phase completely vaporizes and the desired inlet reaction temperature to the reactor is achieved. The fixed bed reactor is charged with $2 \mathrm{~g}$ of the catalyst; the temperature through its longitudinal axis can be monitored and controlled. The height to diameter ratio of the fixed bed is $c a .2: 1$. The average pressure within the reactor is set to 8 bar. The pressurized stream is first cooled in an air condenser and then further cooled in a water cooled-separator. The liquid is separated and may be discharged (e.g. for sampling purposes), and the gas stream returns to the back pressure regulator valve. From there, the gas stream is sent to a vent or sampled for analysis. Analysis of the liquid or gas samples was performed by gas chromatography (CP-3800 GC Agilent) using a CP-Wax 52 CB column.

\section{Results and discussion}

\subsection{Catalyst characterization}

The designation of the samples is presented in Table 1. It should be noted that when using oxalic acid concentrations greater than $1 \mathrm{M}$, the extent of dealumination remained constant $(\mathrm{Si} / \mathrm{Al}<20)$, while the crystallinity was detrimentally affected. Accordingly, only sample DA1 (representative of the oxalic acid-treated samples) is listed in Table 1. It is observed that AHFS enabled dealumination of the parent ZSM- 5 zeolite in a large span of Si/Al molar ratios from 17.9 to 43.1. Fig. 2 shows the XRD analysis of samples DA0 to DA4. It is observed that all the samples possess high crystallinity. Using an AHFS solution with a molarity of $0.1 \mathrm{M}$, it was possible to dealuminate the samples to higher $\mathrm{Si} / \mathrm{Al}$ ratios by simply increasing the time to more than 160 min. However, X-ray diffraction (XRD) analysis revealed that the crystallinity of the samples was severely damaged. Samples DA0 to DA4 are all protonated zeolite 


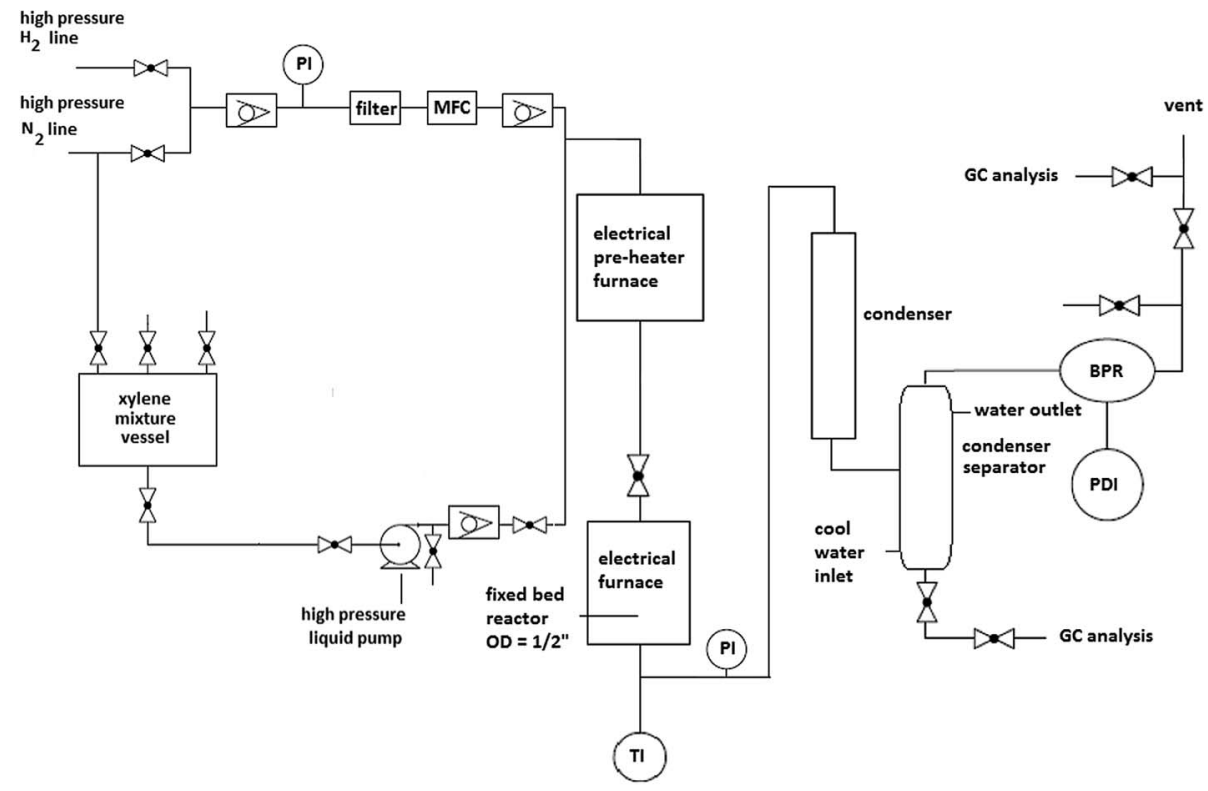

Fig. 1 Flow diagram of the reaction system used for the isomerization studies.

Table 1 Classification of the samples according to the preparatory method and the corresponding chemical analysis (as wt\%) and Si/Al molar ratios based on XRF analysis

\begin{tabular}{|c|c|c|c|c|c|c|c|c|c|c|c|c|}
\hline Sample & $\begin{array}{l}\text { Reagent used for } \\
\text { dealumination/molarity }\end{array}$ & Time (min) & $\mathrm{Si} / \mathrm{Al}$ & $\mathrm{SiO}_{2}$ & $\mathrm{Al}_{2} \mathrm{O}_{3}$ & $\mathrm{Fe}_{2} \mathrm{O}_{3}$ & $\mathrm{CaO}$ & $\mathrm{Na}_{2} \mathrm{O}$ & $\mathrm{K}_{2} \mathrm{O}$ & $\mathrm{MgO}$ & $\mathrm{TiO}_{2}$ & $\mathrm{MnO}$ \\
\hline DA0 & - & - & 17.9 & 88.81 & 4.21 & 0.05 & 0.19 & 0.03 & 0.03 & 0.03 & 0.045 & 0.013 \\
\hline DA2 & AHFS/0.025 & 120 & 28.00 & 89.52 & 2.71 & 0.21 & 0.14 & 0.01 & 0.02 & 0.03 & 0.031 & 0.007 \\
\hline DA3 & AHFS/0.100 & 120 & 41.37 & 93.11 & 1.91 & 0.12 & 0.08 & 0.01 & 0.01 & 0.02 & 0.021 & 0.009 \\
\hline DA4 & AHFS/0.100 & 160 & 43.12 & 92.48 & 1.82 & 0.11 & 0.09 & 0.02 & 0.02 & 0.02 & 0.019 & 0.01 \\
\hline
\end{tabular}

samples with very low contents of alkali or alkaline earth metals (Table 1).

Fig. 3 shows the FTIR spectra of the parent and dealuminated samples. The wave numbers belonging to the main framework T-O asymmetric stretch vibrations (1098.0 to 1100.5 $\mathrm{cm}^{-1}$ ) show a small but orderly shift towards larger numbers. This is in accordance with a report by Triantafillidis et al. ${ }^{11}$ This phenomenon has been theoretically explained to be a consequence of the increase of the number of $\mathrm{Si}-\mathrm{O}$ bonds with respect to $\mathrm{Al}-\mathrm{O}$ bonds. Si-O bonds require higher energy (wave number) for vibration due to the larger atomic mass of Si with respect to $\mathrm{Al}^{12}$ As far as the peak position is concerned, symmetric stretch vibrations of tetrahedral $\mathrm{T}$ atoms are not affected by dealumination treatment $\left(789.0\right.$ to $\left.798.5 \mathrm{~cm}^{-1}\right)$. However, the samples dealuminated with AHFS (DA2 and DA3) show clear increases in the relative integrated intensities of the corresponding peak. A peculiarity arises in the FTIR spectra of the DA2 and DA3 samples. The peaks centered at $1406.0 \mathrm{~cm}^{-1}$ (DA2) and $1405.0 \mathrm{~cm}^{-1}$ (DA3) are unusual. To our knowledge, there are no specific reports of these peaks for H-ZSM-5 zeolites in the available literature. These peaks pertain to samples treated with the highest AHFS molarity. A small probability exists that due to the use of ammonium hexafluorosilicate as the dealumination reagent, some residual $(\mathrm{NH})^{2-}$ groups are present. The latter groups have been reported to exhibit bending and stretching vibration modes in the wave number span of 1402 to $1405 \mathrm{~cm}^{-1}$. ${ }^{13,14}$ However, peaks centered near $1400 \mathrm{~cm}^{-1}$ have been reported for ZSM-5 zeolite produced without organic templates without any assignment. ${ }^{15}$ Samples DA2 and DA3 show a distinct peak at $3657 \mathrm{~cm}^{-1}$ which, based on the reported literature, may belong to $\mathrm{Al}(\mathrm{OH})$ extra framework groups. ${ }^{2,16}$ The same peak is present in the spectrum of the sample dealuminated with oxalic acid (DA1), although it is less pronounced. This attribution requires a complementary confirming analysis, such as ${ }^{27} \mathrm{Al} \mathrm{NMR}$ analysis, and will be discussed later in the text. A peak centered at about $3746.0 \mathrm{~cm}^{-1}$ is observed for the DA1 sample. The same peak also exists in the spectra of the DA2 and DA3 samples, although it is less intense and appears as a shoulder. This peak may be attributed to isolated terminal silanol groups residing on the external surface of the zeolite crystals., ${ }^{2,17}$

Fig. $4 \mathrm{a}-\mathrm{c}$ show the nitrogen adsorption/desorption isotherms for the parent, DA1 and DA3 samples. It may be observed that dealumination does not impart any significant 


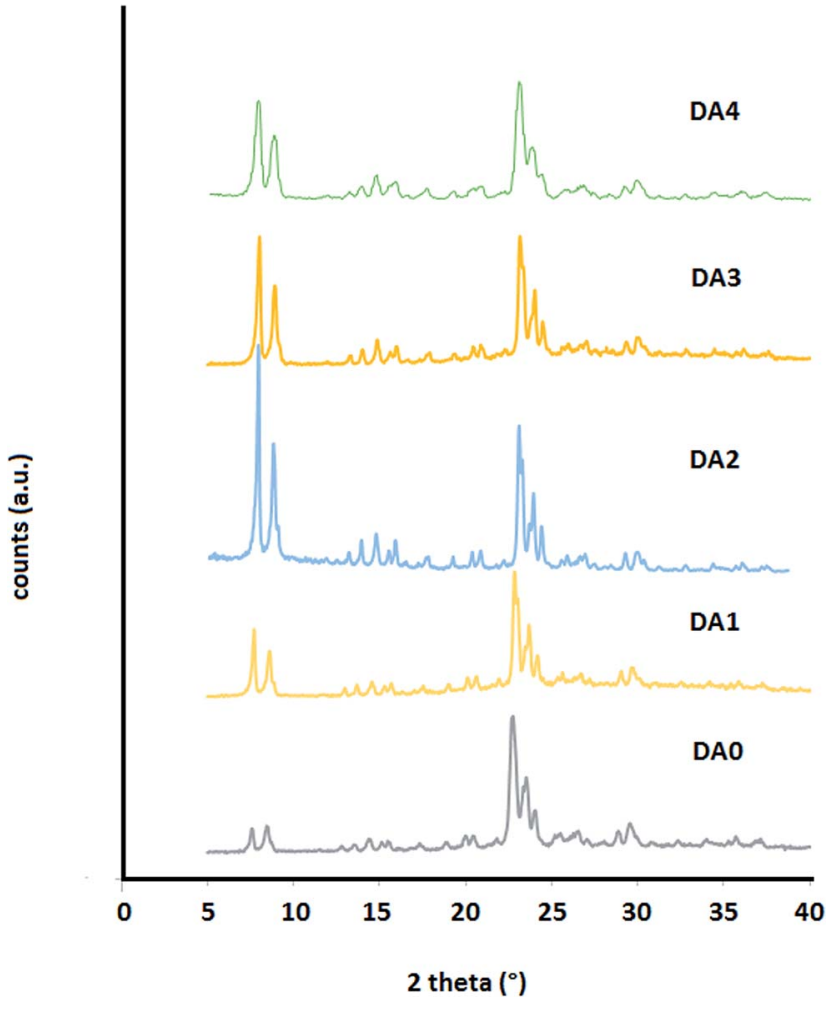

Fig. 2 XRD spectra of samples DAO to DA4.

change in the curves. The quantitative interpretation of the results is presented in Table 2. Changes in specific surface area, micropore volume and external area are minimal. However, gradual increasing trends are observed for the micro and mesoporosity, accompanied by a continuous but slight increase in the total specific surface area. Accordingly, this treatment does not result in any significant change in the pore microstructure. This may be confirmed by referring to the HRTEM pictures of the DA1 and DA3 samples. Fig. 5a illustrates the TEM picture of the parent zeolite, showing its polycrystalline nature. Fig. 5b and $\mathrm{c}$ show the HRTEM pictures of the DA1 and DA3 samples, respectively. It can be clearly observed that the atomic rows of the DA1 sample have a high spatial order; any induced micro or mesoporosity due to aluminum leaching is difficult to detect. Sample DA3 exhibits a slightly less homogenous microporosity. These effects are in accordance with the nitrogen adsorption/ desorption analysis results.

Fig. 6 shows the $\mathrm{NH}_{3}$-TPD analysis results in terms of total and deconvoluted spectra. The DA0, DA1 and DA3 samples show 3,4 and 5 distinct peaks, respectively. The relative integrated intensities of each peak are quantified in Table 3. Generally, the peak maximum intensity of weak acid sites appears in the temperature range of $104{ }^{\circ} \mathrm{C}$ to $121^{\circ} \mathrm{C}$. Medium acid sites appear in the $200{ }^{\circ} \mathrm{C}$ to $215{ }^{\circ} \mathrm{C}$ temperature range. Strong acid sites appear in a wide range of $415^{\circ} \mathrm{C}$ to $650^{\circ} \mathrm{C}$. The latter sites may be classified as type I $\left(415^{\circ} \mathrm{C}\right.$ to $\left.429^{\circ} \mathrm{C}\right)$, type II $\left(570{ }^{\circ} \mathrm{C}\right)$ and type III $\left(650^{\circ} \mathrm{C}\right)$. The parent zeolite exhibits only type I strong acid sites with a relative content of $23.9 \%$. The DA1 sample (dealuminated with oxalic acid) exhibits all three types

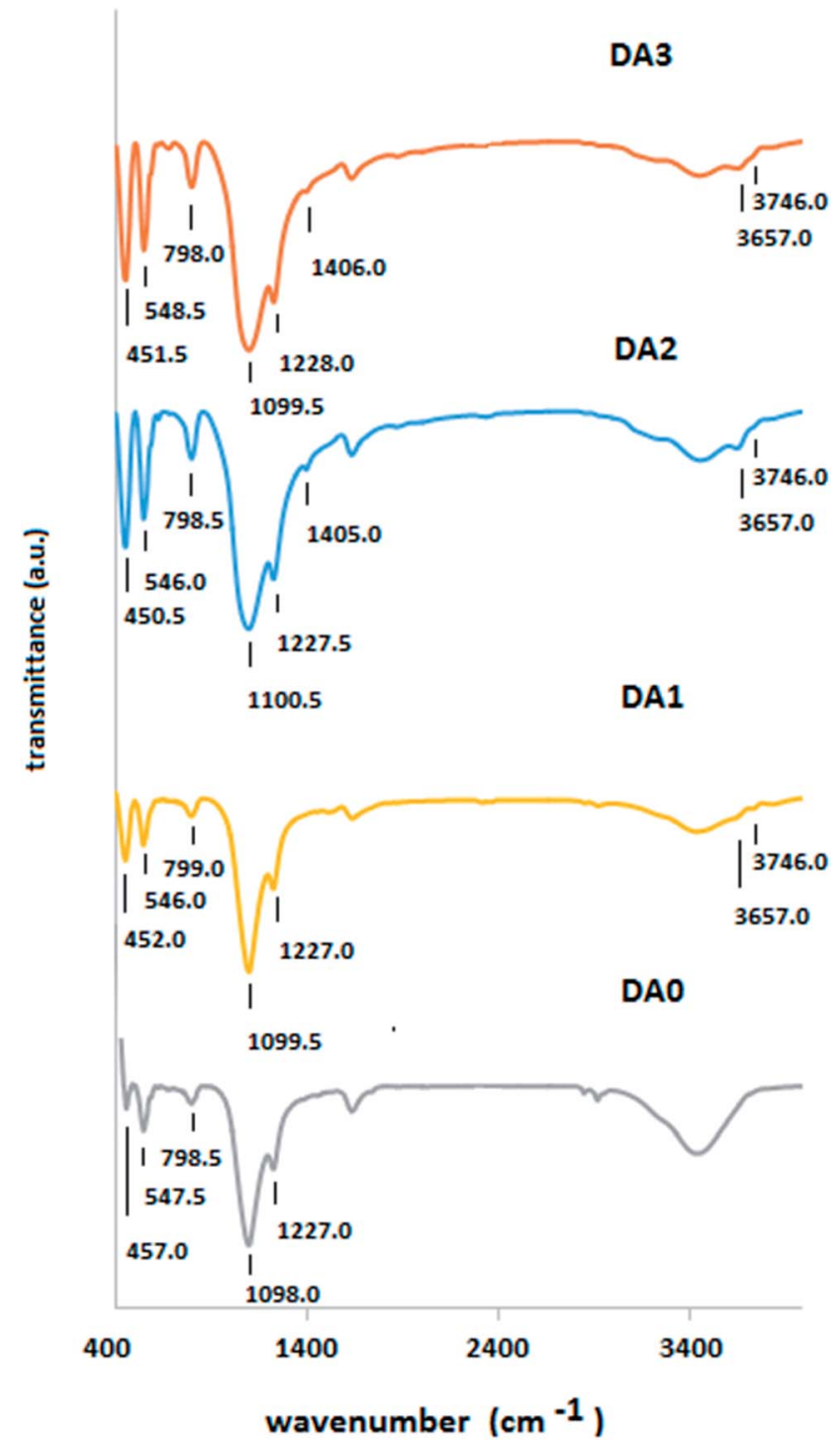

Fig. 3 FTIR spectra of the parent and dealuminated samples.

of strong acid sites: I (24.1\%), II (5.2\%) and III (0.4\%). The DA3 sample (dealuminated with AHFS) exhibits strong acid sites of types I (30.6\%) and III (9.0\%). In addition, referring to Fig. 6, it may be observed that the total absolute integrated intensity increases slightly from the parent to the oxalic acid dealuminated sample. This increase has been reported by Brunner et al. in the case of mild dealumination of ZSM-5 through steaming. ${ }^{18}$ Meanwhile, dealumination using AHSF (sample DA3) results in a reduction of the total acidity. This last finding is rational when we consider the much lower aluminum content of this zeolite sample.

Published studies on the $\mathrm{NH}_{3}$-TPD analysis of hierarchical dealuminated H-ZSM-5 zeolites and H-ZSM-5 zeolites originally synthesized with different Si/Al molar ratios in a span of 27 to 280 report the existence of type II acid sites with peaks centered at temperatures near or less than $450{ }^{\circ} \mathrm{C} . .^{17,19-24}$ The profile containing three types of strong acid sites observed for the oxalic acid-treated sample is novel for protonated ZSM-5 

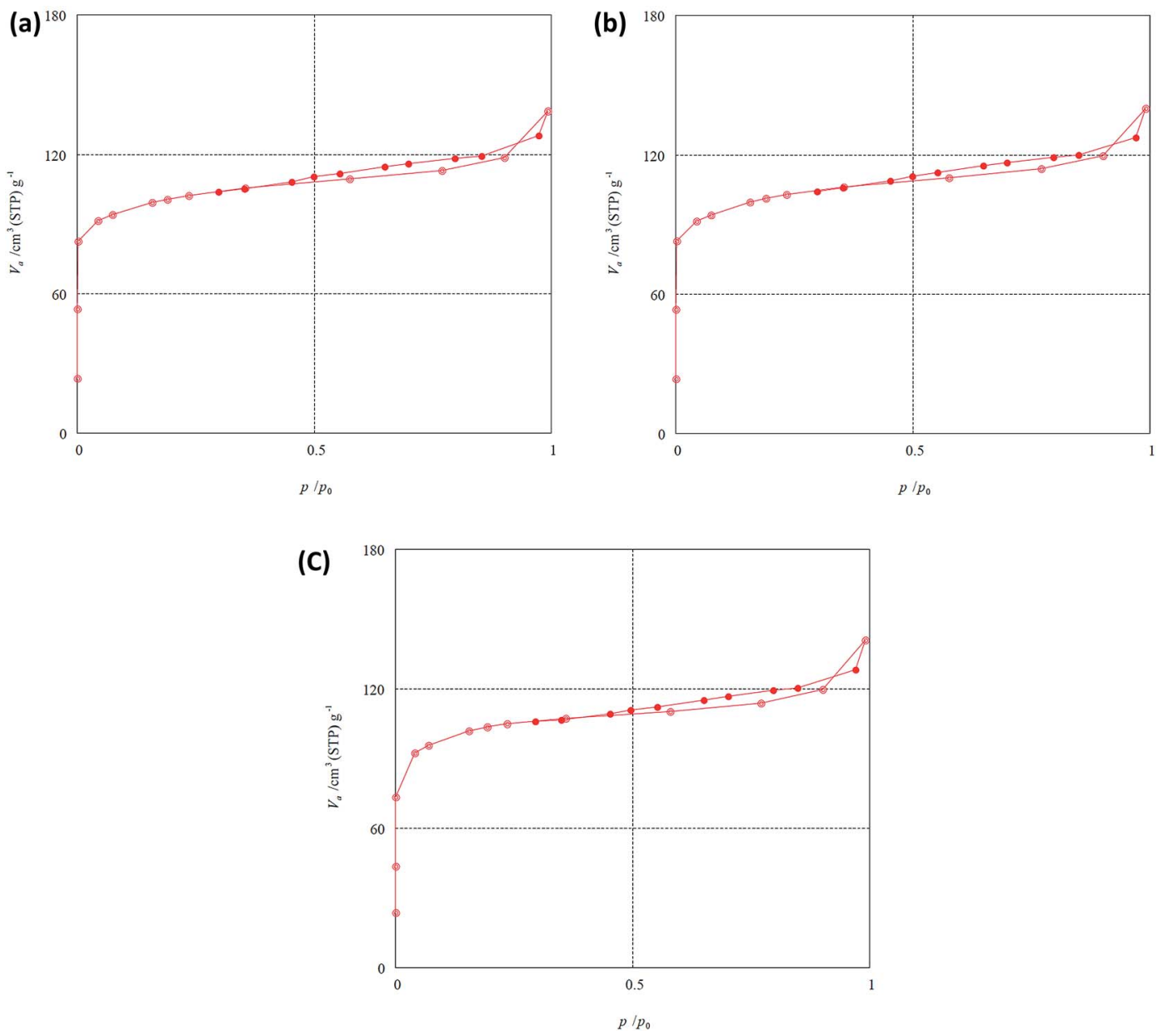

Fig. 4 Nitrogen adsorption/desorption isotherms for the (a) parent, (b) DA1 and (c) DA3 samples.

Table 2 Quantitative results obtained from the nitrogen adsorption/desorption isotherms of the parent and dealuminated samples

\begin{tabular}{lllllll}
\hline Sample & $\begin{array}{l}\text { Specific surface } \\
\text { area }\left(\mathrm{m}^{2} \mathrm{~g}^{-1}\right)\end{array}$ & $\begin{array}{l}\text { Micropore surface } \\
\text { area }\left(\mathrm{m}^{2} \mathrm{~g}^{-1}\right)\end{array}$ & $\begin{array}{l}\text { External surface } \\
\text { area }\left(\mathrm{m}^{2} \mathrm{~g}^{-1}\right)\end{array}$ & $\begin{array}{l}\text { Pore volume } \\
\left(\mathrm{cm}^{3} \mathrm{~g}^{-1}\right)\end{array}$ & $\begin{array}{l}\text { Micropore volume } \\
\left(\mathrm{cm}^{3} \mathrm{~g}^{-1}\right)\end{array}$ \\
\hline DA0 & 380.8 & 358.15 & 22.68 & 0.2142 & 0.1524 & $\begin{array}{l}\text { Mesopore volume } \\
\left(\mathrm{cm}^{3} \mathrm{~g}^{-1}\right)\end{array}$ \\
DA1 & 381.2 & 358.21 & 23.03 & 0.2162 & 0.1532 & 0.0618 \\
DA3 & 389.0 & 366.96 & 22.08 & 0.2175 & 0.1537 & 0.0630 \\
\end{tabular}

zeolites containing almost no ion-exchanged or extraframework metals. It should be recalled that the presence of metals (such as $\mathrm{Cr}$ and V) may create strong acid sites exhibiting peaks centered at about $600{ }^{\circ} \mathrm{C} .{ }^{25,26}$

The AHFS-treated sample (DA3) shows an appreciable content of the strongest acid sites of type III (8.9\%). Referring to the FTIR results, it was mentioned that the peak observed at $3675 \mathrm{~cm}^{-1}$ may belong to extraframework aluminum. Ghasemian et al. traced super acids due to oxo-aluminum species (due to extraframework aluminum) in dealuminated clinoptilolite zeolite from the high temperature $\mathrm{NH}_{3}$-TPD peaks observed at $700{ }^{\circ} \mathrm{C}$ to $750{ }^{\circ} \mathrm{C} .{ }^{27}$ However, the detailed work by Triantafillidis et al. on the dealumination of ZSM- 5 zeolites with AHFS shows that this reagent produces samples free of extraframework aluminum. ${ }^{11}$ Our ${ }^{27} \mathrm{Al}$ MAS NMR analysis also confirms this fact and will be discussed later. Accordingly, it may be stated that the $3657 \mathrm{~cm}^{-1}$ FTIR peak is related to strong bridging hydroxyls at Bronsted sites within the framework.

Fig. 7a and $b$ illustrate the results of ${ }^{27} \mathrm{Al}$ MAS NMR analysis for the DA0, DA1, DA2 and DA3 samples. First of all, it should be stressed out that the original commercial H-ZSM-5 sample contains extraframework aluminum of octahedral character. Treatment with oxalic acid cannot eliminate extraframework residual aluminum. This is generally an arguable matter as far as the oxalic acid molarity used for sample DA1 is concerned. At this molarity, highly soluble $\left[\mathrm{Al}\left(\mathrm{C}_{2} \mathrm{O}_{4}\right)_{3}\right]^{3-}$ complexes are prevalent and highly stable. ${ }^{28}$ In other words, extraframework aluminum may be preferentially chelated. Incomplete egress of 
(a)

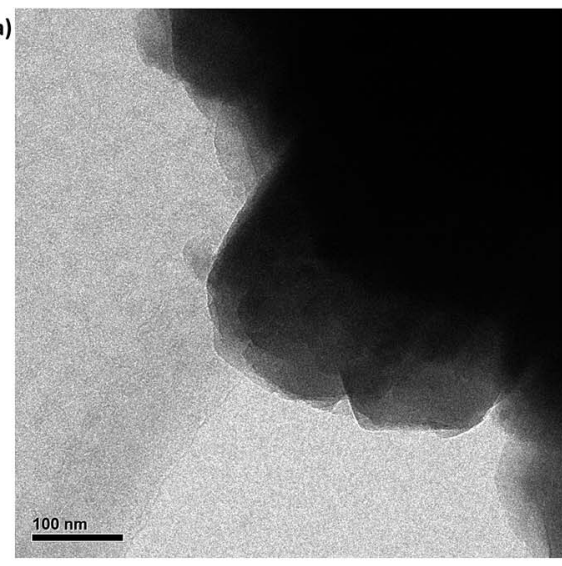

(b)

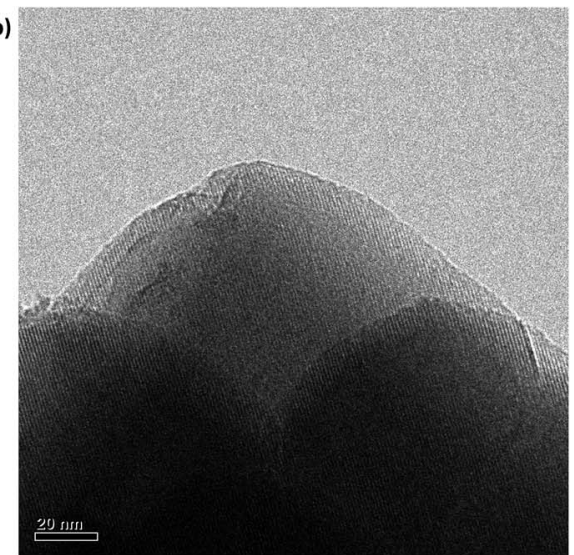

(c)

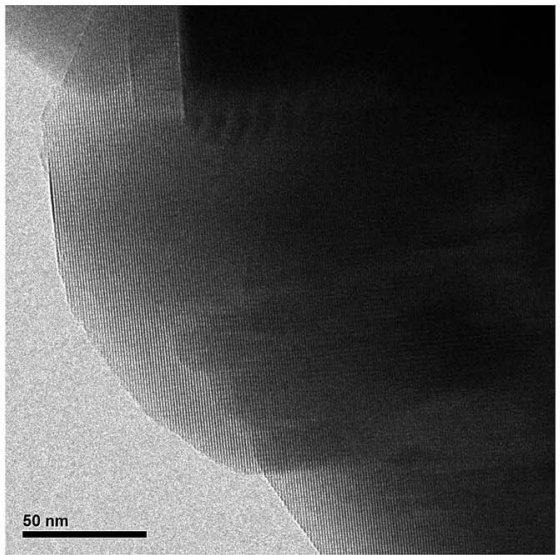

Fig. 5 HRTEM pictures of the (a) parent, (b) DA1 and (c) DA3 samples.

these aqueous complexes during the isolation/washing steps might be the cause of the residual extraframework aluminum observed.

A more attentive analysis reveals the slight growth of a peak around 70 ppm upon dealumination with oxalic acid. This is indicated by an arrow in Fig. 7a and is attributed to tetrahedral aluminum atoms with a different coordination (with respect to the majority of tetrahedral aluminum atoms with a resonance appearing at ca. $50 \mathrm{ppm}$ ). It may be argued that this is a noise peak. However, evidence presented by other researchers places this suggestion under question. Sklenak et al. discuss these different sites of aluminum atoms in ZSM-5 pentasile zeolites. ${ }^{29}$ Hierarchical ZSM-5 zeolites produced by rectorite (a natural layered aluminosilicate) in the absence of secondary mesoscale templates also show this peculiarity in the corresponding ${ }^{27} \mathrm{Al}$ MAS NMR spectra. ${ }^{20}$ Therefore, it may be stated that treatment with oxalic acid creates a small minority of differently coordinated tetrahedral aluminum atoms. Based on Fig. 7b, it can be clearly observed that AHFS has almost completely eliminated any extraframework aluminum, as expected according to the literature. ${ }^{\mathbf{1 1}}$

\subsection{Catalytic study of the ethylbenzene/xylene mixture isomerization reaction}

Before considering the pertinent experimental results, some terms should be defined:

$$
\begin{gathered}
\mathrm{EB}_{\mathrm{C}}(\%) \equiv \text { ethylbenzene conversion }(\%) \\
\equiv\left(1-\frac{C_{\text {ethylbenzne,product }}}{C_{\text {ethylbenzene,feed }}}\right) \times 100 \\
\text { p-Xylene a.t.e. }(\%) \equiv \frac{C_{\text {para } \text { xylene,product }}-C_{\text {para } \text { xylene,feed }}}{C_{\text {para xylene,equilibrium }}(T)-C_{\text {para } \text { xylene,feed }}} \\
\times 100 \\
\mathrm{C}_{8} \text {-aromatics loss }(\%) \equiv\left(1-\frac{C_{\mathrm{C}_{8} \text {-aromatics,product }}}{C_{\mathrm{C}_{8} \text {-aromatics,feed }}}\right) \times 100
\end{gathered}
$$

where $C_{i}$ is the concentration (wt\%) of component $i$ in the feed or product and $T$ is the reaction temperature.

Fig. 8 shows the $p$-xylene a.t.e. as a function of weight hourly space velocity (WHSV) and the reaction temperatures for samples DA0, DA1, DA2, DA3 and DA4. Generally, it is observed that dealumination has a pronounced and positive effect on the value of $p$-xylene a.t.e. at $390{ }^{\circ} \mathrm{C}$ and $400{ }^{\circ} \mathrm{C}$. At $380{ }^{\circ} \mathrm{C}$, the highly dealuminated samples DA3 and DA4 show poorer performance with respect to the parent zeolite sample. Interestingly, the catalytic behavior is quite similar for the DA1 (oxalic acidtreated) and DA2 (mildly AHFS-treated) zeolite samples. Both of these can increase $p$-xylene a.t.e. by more than $10 \%$ at a reaction temperature of $400{ }^{\circ} \mathrm{C}$ and a WHSV equal to $2 \mathrm{~h}^{-1}$ compared to the parent zeolite under the same operating 


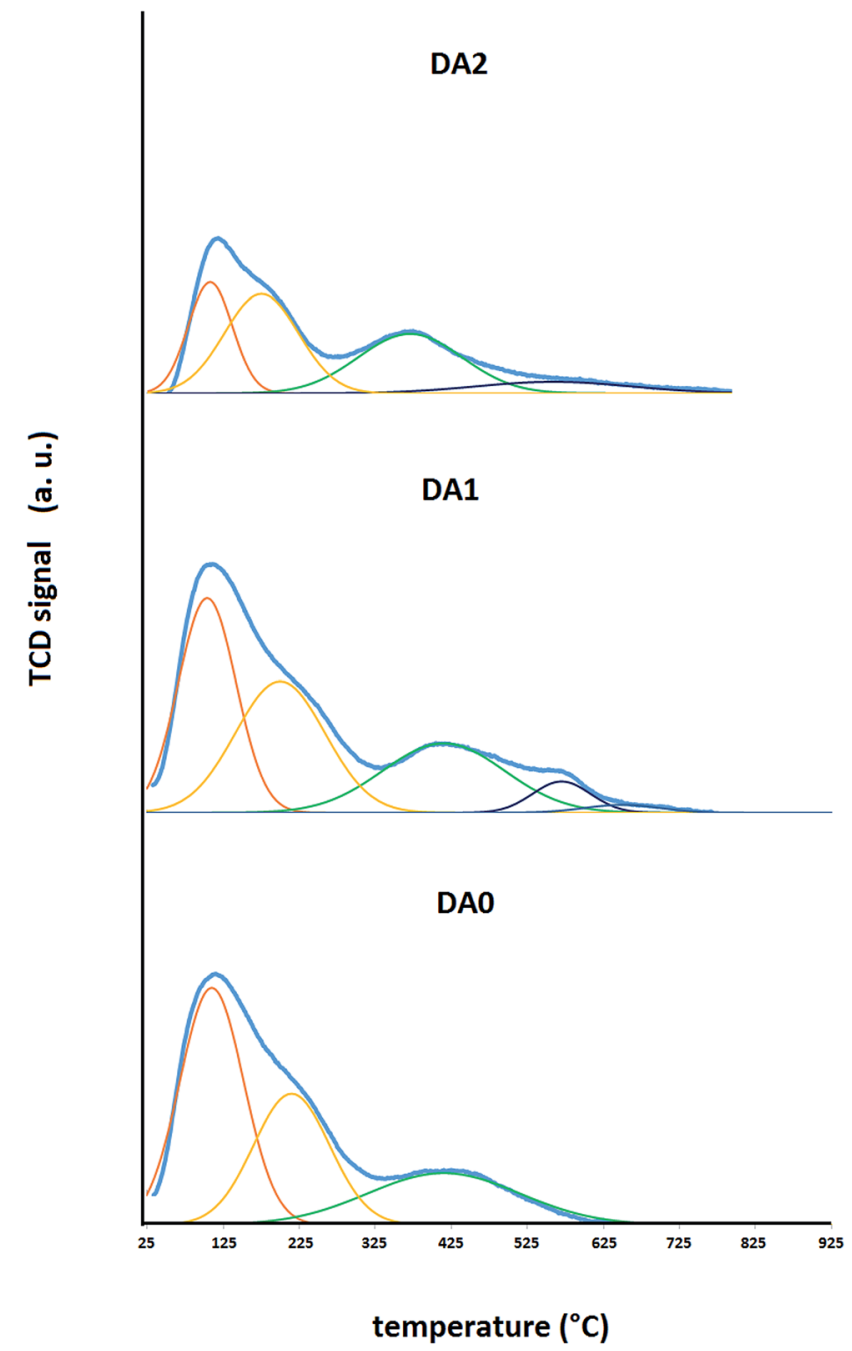

Fig. $6 \mathrm{NH}_{3}-\mathrm{TPD}$ analysis in terms of total and deconvoluted spectra for the DA0, DA1 and DA3 samples.

conditions. It should be recalled that, regarding the thermodynamics of the reaction system, a temperature increase should result in a decrease in $p$-xylene a.t.e. ${ }^{30}$ The trend of $p$-xylene a.t.e. change versus temperature increase at constant WHSV generally increases. Accordingly, it may be stated that the overall reaction kinetics is controlled by diffusional mass transfer of the reacting components within the zeolite micropores.

Fig. 9 shows the $\mathrm{EB}_{\mathrm{C}}$ of the different samples as a function of WHSV and reaction temperature. An interesting result is clearly observed. The DA1 sample (oxalic acid-treated) shows distinct behavior; $\mathrm{EB}_{\mathrm{C}}$ increases by more than $30 \%$ with respect to the parent zeolite at all the reaction temperatures and WHSVs under consideration. Meanwhile, the other dealuminated samples exhibit reduced $\mathrm{EB}_{\mathrm{C}}$ values compared to the parent sample. In the case of AHFS-treated samples, the more the zeolite is dealuminated, the lower the resulting $\mathrm{EB}_{\mathrm{C}}$. Increase of temperature is accompanied with an increase of $\mathrm{EB}_{\mathrm{C}}$ for all the samples (at constant WHSV).

Finally, the $\mathrm{C}_{8}$-aromatics loss is considered (Fig. 10). The sample treated with oxalic acid (DA0) shows distinct behavior at 


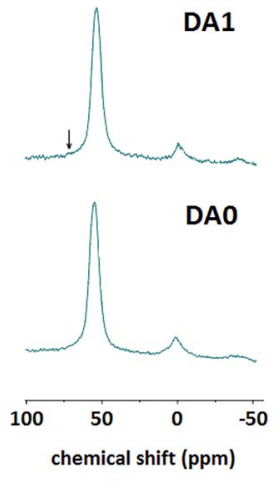

(a)

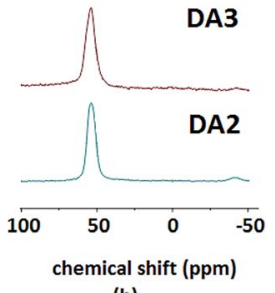

(b)

Fig. $7{ }^{27}$ Al MAS NMR analysis of the (a) DAO and DA1 and (b) DA2 and DA3 samples. A unique framework tetrahedral Al resonance is indicated by an arrow.
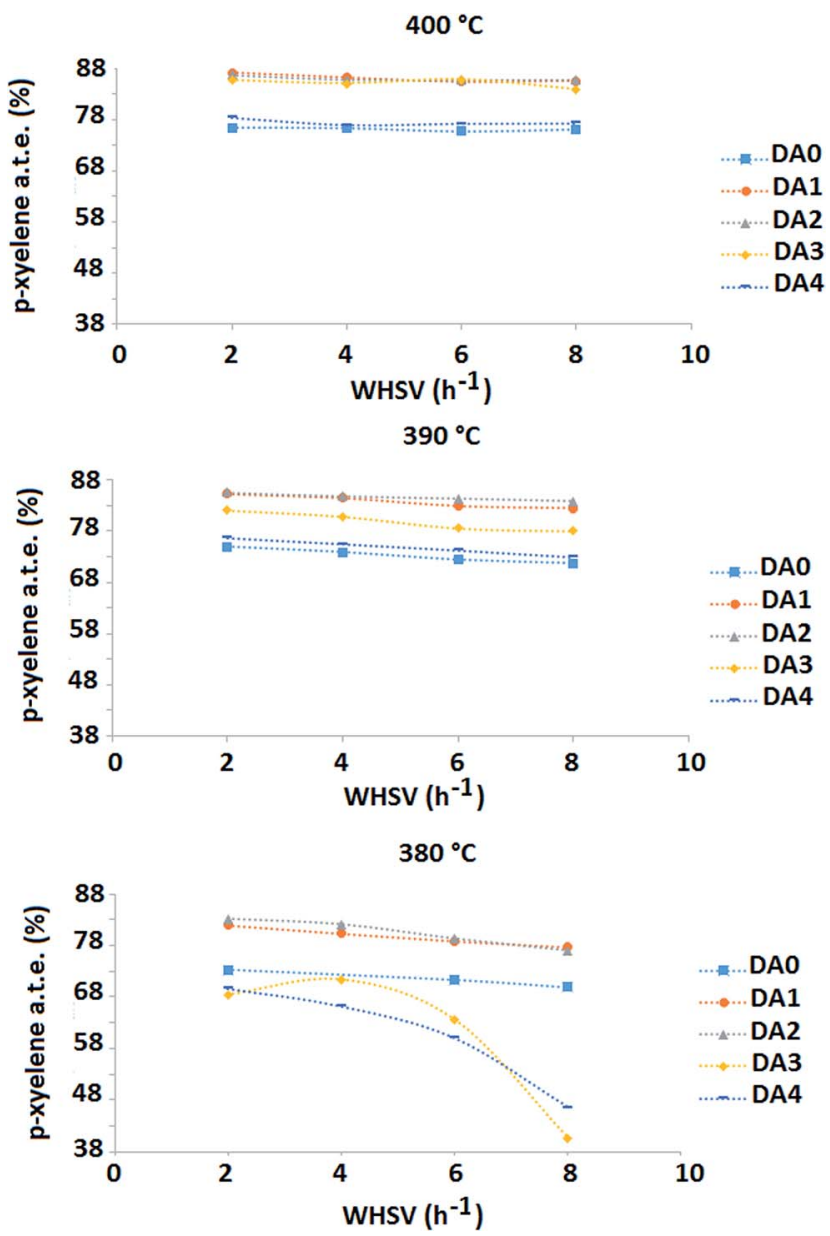

Fig. 8 -Xylene a.t.e. as a function of WHSV, reaction temperature and treatment conditions.

all the reaction temperatures under investigation. Its $\mathrm{C}_{8^{-}}$ aromatics loss is more than double that of the other samples (at constant $T$ and WHSV) and is not significantly temperature dependent. It changes between 10.0 and $11.5 \mathrm{wt} \%$ over the whole temperature range of $380{ }^{\circ} \mathrm{C}$ to $400{ }^{\circ} \mathrm{C}$. Sample DA2
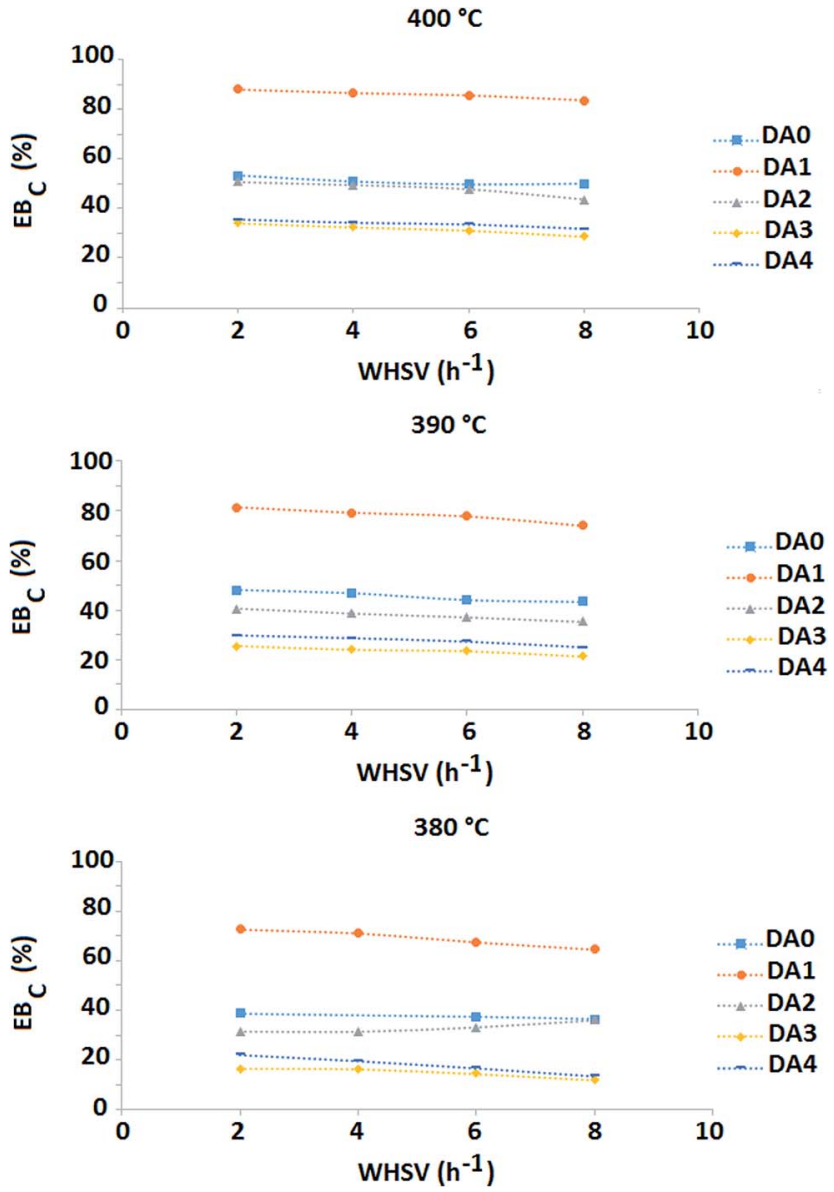

Fig. $9 \mathrm{~EB}_{\mathrm{C}}$ as a function of WHSV, reaction temperature and treatment conditions.

results in $\mathrm{a}_{8}$-aromatics loss between $3.6 \%$ and $4.0 \%$ at $380{ }^{\circ} \mathrm{C}$, which increases to a range of $4.3 \%$ to $4.8 \%$ at $390{ }^{\circ} \mathrm{C}$ and further increases to $5.3 \%$ to $6.2 \%$ at $400{ }^{\circ} \mathrm{C}$. The $\mathrm{C}_{8}$-aromatics loss of the parent zeolite sample lies between $4.4 \%$ and $5.6 \%$ throughout the whole temperature range. Sample DA3 shows the lowest $\mathrm{C}_{8^{-}}$aromatics loss (1.9\% to $3.4 \%)$.

In order to present a theoretical explanation of the catalytic behavior of the different samples, the ethylbenzene reactions are considered first. Ethylbenzene may undergo isomerization, disproportionation and dealkylation reactions. Disproportionation of ethylbenzene results in the formation of diethylbenzene isomers and benzene. Accordingly, it is informative to monitor the trend of benzene formation throughout the experiments (Fig. 11). It was observed that the concentration of benzene in the product is nearly nil for the parent zeolite at all the reaction temperatures under consideration. Instead, the samples DA1 and DA2 result in substantial production of benzene. For the latter samples, benzene concentration increases with increasing temperature. Based on the previously described experimental results, it may be stated that samples DA1 and DA2 promote ethylbenzene disproportionation. The sample treated with oxalic acid (DA1) exhibits the largest tendency for the latter reaction. As discussed in the previous section, dealumination with oxalic acid did result in slight 

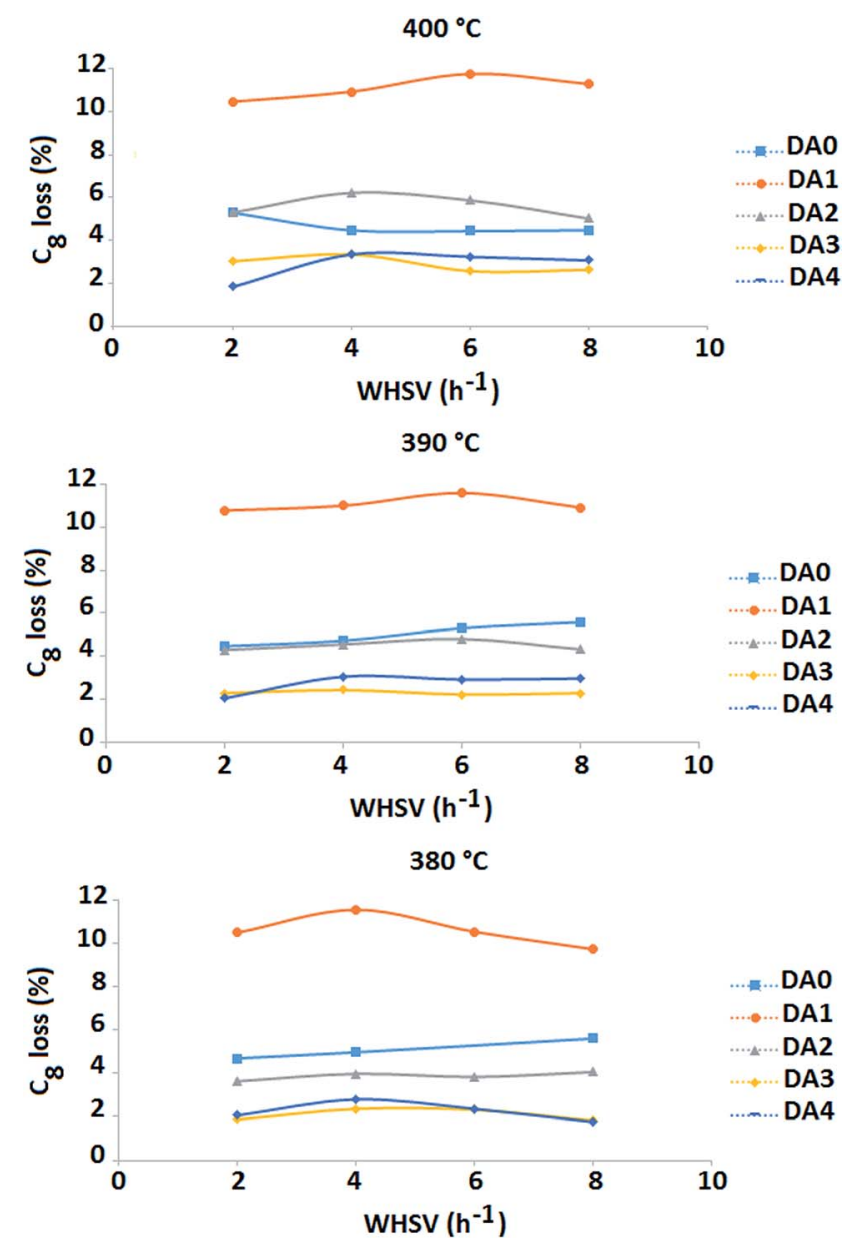

Fig. $10 \mathrm{C}_{8}$-aromatics loss as a function of WHSV, reaction temperature and treatment conditions.

increases in the microporosity and specific surface area. Based on the $\mathrm{NH}_{3}$-TPD results, this was accompanied by a substantial increase of total acidity and the creation of stronger acid sites compared to the parent zeolite. Both of these effects may enhance the ethyl benzene disproportionation reaction. The effect is less pronounced for the samples dealuminated with AHFS, as their total acidity is significantly less than that of sample DA1 due to their higher $\mathrm{Si} / \mathrm{Al}$ ratios. A trade-off appears to exist between increased microporosity and reduced total acidity in the case of the samples treated with AHFS. Added microporosity through dealumination presumably allows ethylbenzene to disproportionate through a variety of reaction mechanisms involving bulky intermediates that are primarily prohibited by the parent H-ZSM-5 zeolite. ${ }^{10}$ Elucidation of the reaction mechanism in terms of the new micropore architectures and acid site strength distributions is a subject of debate and requires a separate study.

The substantial enhancement of para xylene selectivity observed for the DA1 and DA2 samples is worthy of consideration. As discussed in the previous paragraph, the micropore structure and channel interactions of the parent zeolite as a function of dealumination were only moderately altered. However, this modification was very effective. The kinetic
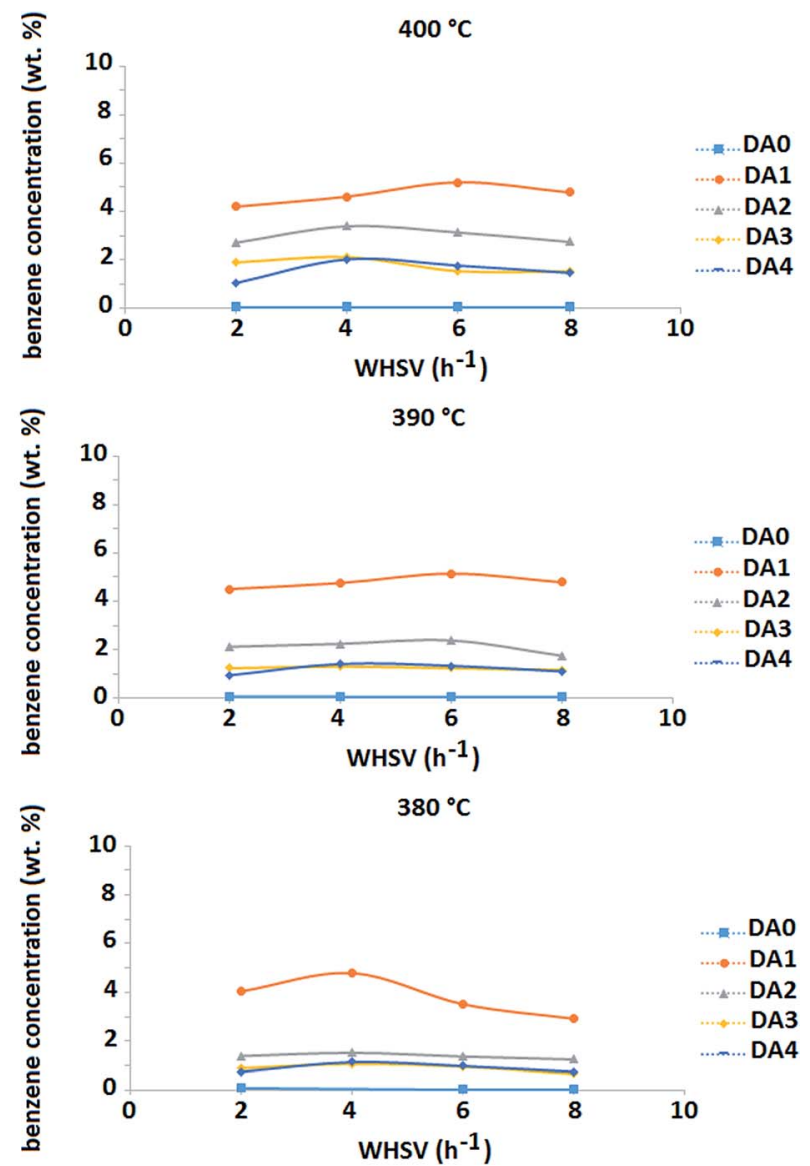

Fig. 11 Benzene concentration as a function of WHSV, reaction temperature and treatment conditions.

diameters of ortho, meta and para xylenes have been reported as 6.8, 6.8 and $5.8 \AA$, respectively. ${ }^{31}$ These are close to the sinusoidal $\left(5.1 \times 5.5 \AA^{2}\right)$ and straight $\left(5.4 \times 5.6 \AA^{2}\right)$ channel pore diameters of the parent H-ZSM-5 zeolite. Although the intersections of the perpendicular channels form cavities with diameters as large as $8.6 \AA$, a general consensus exists that xylene isomerization reactions proceed by a unimolecular pathway mechanism where bulky intermediates such as trimethylbenzene molecules are not involved. ${ }^{31}$ Accordingly, the authors of the present work presume that the dealuminated

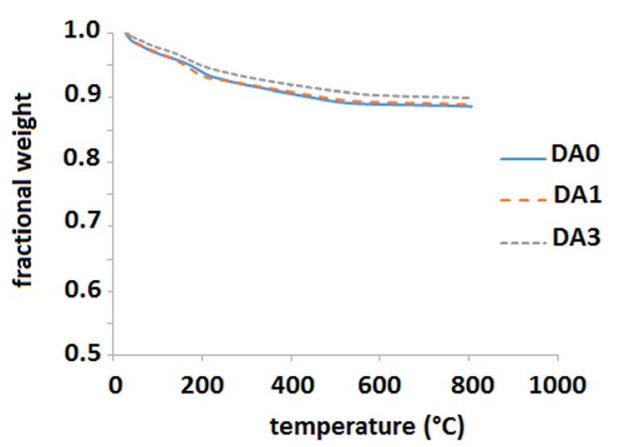

Fig. 12 TGA analysis of the spent DAO, DA1 and DA3 samples after ca. $12 \mathrm{~h}$ on stream. 
samples DA1 and DA2 undergo a slight increase in average pore diameter, facilitating the entry of ortho and meta isomers into the pore labyrinth and augmenting the velocity of egress of the para isomer from the pores. However, excess enlargement of the average pore diameter is detrimental and may have the opposite effect (samples DA3 and DA4 at $380^{\circ} \mathrm{C}$ ). To our knowledge, this improvement in para xylene selectivity by simple dealumination has not been reported previously. The more remarkable point is that this enhancement may be tailored to be accompanied with high ethylbenzene conversion through disproportionation (oxalic acid treatment) or not (AHFS treatment).

One final point is remarkable. The zeolites that are the subject of this study showed very low deactivation rates. To be more precise, our experiments showed that all the zeolite samples retained their catalytic characteristics within 1\% error up to $12 \mathrm{~h}$ (the maximum time on stream applied). However, this does not mean that they do not undergo deactivation. In fact, they are deactivated at early stages in less than $2 \mathrm{~h}$ and reach a quasi-equilibrium coke content. Product analysis was performed after this preliminary period. Afterwards, no detectable deactivation occurred up to $12 \mathrm{~h}$ on stream. Fig. 12 shows the TGA results for the spent parent, DA1 and DA3 zeolite samples after $c a .12 \mathrm{~h}$ on stream. The thermograms are very similar. If we attribute the weight loss after $400{ }^{\circ} \mathrm{C}$ to coke burning, the amount of deposited coke is less than $1.5 \mathrm{wt} \%$. It should be mentioned that the slow deactivation kinetics is mainly due to the high hydrogen partial pressure used in this series of experiments to mimic the industrial process.

\section{Conclusions}

The influence of ZSM-5 zeolite dealumination on simultaneous catalytic xylene isomerization and ethylbenzene disproportionation reactions has been studied for the first time. Mild increases of the Si/Al molar ratio by one unit using oxalic acid or by 10 units by AHFS results in a more than $10 \%$ increase in para xylene approaching equilibrium at $400{ }^{\circ} \mathrm{C}$ and WHSV $=2 \mathrm{~h}^{-1}$. However, the oxalic acid-treated sample increases ethylbenzene conversion by more than $30 \%$ with respect to the parent zeolite under the applied operating conditions. The AHFS-treated samples, irrespective of the extent of dealumination imparted (Si/Al ratio between 28 and 43), exhibit decreased ethylbenzene conversion compared to the parent zeolite. The results can be explained based on the various characterization techniques applied.

In summary, it may be stated that dealumination of ZSM-5 zeolite with oxalic acid results in an attractive catalyst for petrochemical plants, where high para xylene selectivity and near-complete ethylbenzene dealkylation are desirable.

\section{Acknowledgements}

This work was financed by BIPC, Mahshahr, Iran under the contract number $08-133 / 57666$. BIPC is highly acknowledged for the support provided. The authors offer heartfelt thanks to Navid Naderpour, Rahman Mahmoudi, Majid Mollavali, Amin Moavi, Mohammad Ali Sharifi and Asghar Jamali for their cooperation and the fruitful human ambience they created throughout the work. Bahir Duraki is greatly acknowledged for his help in NMR analysis.

\section{References}

1 S. Kumar, A. K. Sinha, S. G. Hegde and S. Sivasanker, J. Mol. Catal. A: Chem., 2000, 154, 115-120.

2 C. Fernandez, I. Stan, J. P. Gilson, K. Thomas, A. Vicente, A. Bonilla and J. Perez-Ramirez, Chem.-Eur. J., 2010, 16, 6224-6233.

3 C. Ding, X. Wang, X. Guo and S. Zhang, Catal. Commun., 2007, 9, 487-493.

4 R. Rachwalik, Z. Olejniczak and B. Sulikowski, Catal. Today, 2006, 114, 211-216.

5 M. Mueller, G. Harvey and R. Prins, ${ }^{29} \mathrm{Si}$ and ${ }^{27} \mathrm{Al}$ MAS NMR, Microporous Mesoporous Mater., 2007, 34, 135-147.

6 T. C. Tsai, I. Wang, C. K. Huang and S. D. Liu, Appl. Catal., A, 2007, 321, 125-134.

7 W. Hong and D. Olson, US Pat. 3856871 A, 1974.

8 G. Burgfels, J. Schoenlinner and F. Schmidt, US Pat. 7244 409 B2, 2007.

9 Y. F. Chu, F. A. Smith and A. W. Chester, EP 0087906 A1, 1983.

10 H. K. Min and S. B. Hong, J. Phys. Chem., 2011, 115, 1612416133.

11 C. S. Triantafillidis, A. G. Vlessidis, L. Nalbandian and N. P. Evmiridis, Microporous Mesoporous Mater., 2001, 47, 36-388.

12 M. A. Ali, B. Brisdon and W. J. Thomas, Appl. Catal., A, 2003, 252, 149-162.

13 B. Bonelli, M. Armandi, C. O. Arean and E. Garrone, ChemPhysChem, 2010, 11, 3255-3261.

14 X. J. Wang, R. Nesper, C. Villevieille and P. Novák, Adv. Energy Mater., 2013, 3, 606-614.

15 Y. Cheng, L. J. Wang, J. S. Li, Y. C. Yang and X. Y. Sun, Mater. Lett., 2005, 59, 3427-3430.

16 C. Pueker, J. Mol. Struct., 1995, 349, 317-320.

17 B. Puertolas, L. Garcia-Andujar, T. Garcia, M. V. Navarro, S. Mitchell and J. Perez-Ramirez, Appl. Catal., B, 2014, 154155, 161-170.

18 E. Brunner, H. Ernst, D. Freude, T. Froehlich, M. Hunger and H. Pfeifer, J. Catal., 1991, 127, 34-41.

19 Y. Yue, H. Liu, P. Yuan, T. Li, C. Yu, H. Bi and X. Bao, J. Catal., 2014, 319, 200-210.

20 J. Ding, H. Liu, P. Yuan, G. Shi and X. Bao, ChemCatChem, 2013, 5, 2258-2269.

21 J. Zheng, G. Wang, M. Pan, D. Guo, Q. Zhao, B. Li and R. Li, Microporous Mesoporous Mater., 2015, 206, 114-120.

22 S. Narayanan, J. Judith Vijaya, S. Sivasanker, L. John Kennedy and S. K. Jesudoss, Powder Technol., 2015, 274, 338-348.

23 M. Osman, L. Atanda, M. M. Hossain and S. Al-Khattaf, Chem. Eng. J., 2013, 222, 498-511.

24 L. Shirazi, E. Jamshidi and M. R. Ghasemi, Cryst. Res. Technol., 2008, 43, 1300-1306. 
25 T. Ma, H. Imai, M. Yamawaki, K. Terasaka and X. Li, Catalysts, 2014, 4, 116-128.

26 M. Hamidzadeh, M. Ghassemzadeh, A. Tarlani and S. Sahebdel Far, Orient. J. Chem., 2016, 32, 481-490.

27 N. Ghasemian, C. Falamaki, M. Kalbasi and M. Khosravi, Chem. Eng. J., 2014, 252, 112-119.

28 R. Giudici, Diss. ETH No. 13358, 1999.
29 S. Sklenak, J. Dedecek, C. Li, B. Wichterlova, V. Gabova, M. Sierka and J. Sauer, Phys. Chem. Chem. Phys., 2009, 11, 1237-1247.

30 R. D. Chirico and W. V. Steele, J. Chem. Eng. Data, 1997, 42, 784-790.

31 D. H. Olson and W. O. Hang, ACS Symp. Ser., 1984, 248, 275307. 